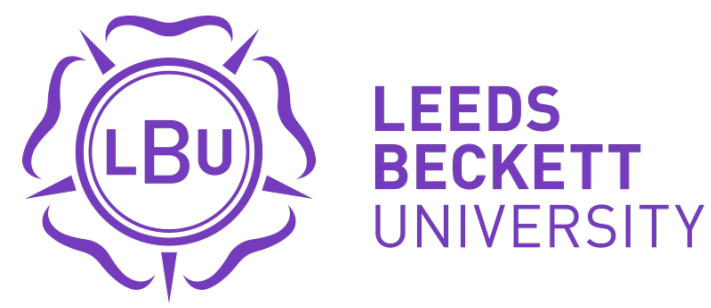

Citation:

Vajpayee, V and Becerra, V and Bausch, $\mathrm{N}$ and Banerjee, S and Deng, J and Shimjith, SR and Arul, AJ (2020) Gain Scheduled Subspace Predictive Control of a Pressurized Water-type Nuclear Reactor. 2020 28th Mediterranean Conference on Control and Automation (MED). pp. 91-96. ISSN 2325-369X DOI: https://doi.org/10.1109/MED48518.2020.9182853

Link to Leeds Beckett Repository record:

https://eprints.leedsbeckett.ac.uk/id/eprint/6982/

Document Version:

Article (Accepted Version)

(C) 2020 IEEE. Personal use of this material is permitted. Permission from IEEE must be obtained for all other uses, in any current or future media, including reprinting/republishing this material for advertising or promotional purposes, creating new collective works, for resale or redistribution to servers or lists, or reuse of any copyrighted component of this work in other works.

The aim of the Leeds Beckett Repository is to provide open access to our research, as required by funder policies and permitted by publishers and copyright law.

The Leeds Beckett repository holds a wide range of publications, each of which has been checked for copyright and the relevant embargo period has been applied by the Research Services team.

We operate on a standard take-down policy. If you are the author or publisher of an output and you would like it removed from the repository, please contact us and we will investigate on a case-by-case basis.

Each thesis in the repository has been cleared where necessary by the author for third party copyright. If you would like a thesis to be removed from the repository or believe there is an issue with copyright, please contact us on openaccess@leedsbeckett.ac.uk and we will investigate on a case-by-case basis. 


\title{
Gain Scheduled Subspace Predictive Control of a Pressurized Water-type Nuclear Reactor
}

\author{
Vineet Vajpayee, Victor Becerra, Nils Bausch, Shohan Banerjee, Jiamei Deng, S. R. Shimjith, A. John Arul
}

\begin{abstract}
This work presents a methodology for designing subspace-based gain scheduled predictive controller for nuclear reactor power control. The main idea is to design a family of predictive controllers directly from measurements and integrate them without employing any explicit process model. The developed controller incorporates the robustness feature of subspace identification with the adaptive capability of gain scheduling in a predictive control set-up. The controller is designed to handle process variations effectively. The efficacy of the proposed controller is demonstrated for load-following transients using a simulated model of a PWR-type nuclear reactor. Simulation results show that the proposed strategy is effective in addressing the load-following control problem of a non-linear parameter-varying PWR nuclear reactor system.
\end{abstract}

\section{INTRODUCTION}

Nuclear power plants (NPP) are complex, highlyconstrained, non-linear systems with time-varying behaviour at different operating power levels. System parameters associated with reactor core, thermal hydraulics, and internal reactivity feedbacks differ significantly with variation in neutronic power. The system response is further deteriorated because of fuel burn-up and other modelling uncertainties. For instance, during load-following operation, routine load cycles can significantly degrade NPP performance due to a broad range of power variations. The control system of an NPP must be able to respond steadily to fast variations in demand power without compromising the performance. The dynamics of an NPP differ significantly across the operating range so a linear controller can not guarantee optimal performance over the entire range. Thus, it is of prime importance to improve the existing reactor power control technique for enhanced safety and operability of NPP.

Model Predictive Control (MPC) is an advanced optimal control design approach that has been practised considerably in industry. MPC has the ability to adapt to new operating conditions and allows simpler constraints handling. It solves an optimization problem to determine future control input over a time period. Traditionally, MPC calls for an accurate

Vineet Vajpayee (vineet.vajpayee@port.ac.uk), Victor Becerra (victor.becerra@port.ac.uk), and Nils Bausch (nils.bausch@port.ac.uk) are with School of Energy and Electronic Engineering, University of Portsmouth, Portsmouth, PO1 3DJ, United Kingdom.

Shohan Banerjee (s.banerjee@leedsbeckett.ac.uk) and Jiamei Deng (j.deng@leedsbeckett.ac.uk) are with School of Built Environment, Engineering, and Computing, Leeds Beckett University, Leeds, LS6 3QS, United Kingdom.

S. R. Shimjith (srshim@barc.gov.in) is with Reactor Control System Design Section, Bhabha Atomic Research Centre, Mumbai, 400 085, India and Homi Bhabha National Institute, Mumbai, 400 094, India.

A. John Arul (arul@igcar.gov.in) is with Probabilistic Safety, Reactor Shielding and Nuclear Data Section, Indira Gandhi Centre for Atomic Research, Kalpakkam, 603 102, India. mathematical model of the process to design the control law [1]. The application of linear or non-linear MPC to the constrained load-following operation of a pressurized watertype reactor (PWR) has engaged vast research interest in the last decade [2]-[5]. For instance, $\mathrm{Na}$ et al. [2] applied linear MPC to the control of power level and axial power distribution. The load-following problem for movable NPP plants is attempted using the linear multiple MPC approach [3]. Eliasi et al. [4] designed a non-linear robust MPC strategy for bounding xenon oscillations.

System description using Linear Parameter Varying (LPV) approach presents a framework to control non-linear systems. For instance, Kothare et al. [6] developed a steam generator level control using the LPV-based MPC approach. The LPV control takes a conservative design approach by forming a strategy based on the worst-case scenario which is prone to poor performance. Implementation of model-based LPV control is challenging as it demands an accurate description of the process in between operating levels. Moreover, such descriptions are not always present or may be difficult to acquire, as in case of nuclear reactor systems.

On the other hand, control strategies based on gain scheduling provide a viable alternative to the LPV control approach [7]. Gain scheduling is a widely exercised control design approach for complex non-linear processes either with time-varying or operating level-dependent behaviours [8]. Gain scheduling is particularly favoured by industries over other non-linear control methods due to its generality, simplicity, and ability to manage control design trade-off [9]. Gain scheduled controllers have been used for steam generator water level control [10]-[12]. The behaviour of a non-linear system can be reasonably described using a family of linearised systems. These linearised systems are evaluated at a set of well-defined equilibrium or operating points. Linear controllers are designed at these points, ensuring that relevant performances are obtained in the proximity of the operating points. These individual controllers are then parametrized and integrated to form a family of controllers to cover the whole operating range of the system. Thus, the resulting controller is implemented as a single controller, whose parameters are varied according to the scheduling variables. In contrast, a weighing mechanism can be employed to use several controllers working in parallel such that the resulting control action is a weighted combination of the control outputs of the individual controllers.

An important limitation of the model-based approach is that it entails an accurate mathematical description of the process a priori. In case of an NPP, variation in reactor 
parameters with operating power level limits the modelbased techniques to guarantee the desired performance. In contrast, data-based approaches such as Subspace Predictive Control (SPC) allow easy adaptation to the time-varying characteristic of the process [13]-[18]. SPC combines the estimation of linear predictor using subspace identification with the formulation of receding horizon control design [13]. Contrary to the classical MPC which first models the process and then estimates the controller parameters, the SPC combines these two steps into one, thereby reducing the computation time, complexity, and errors arising due to model-plant mismatch. Furthermore, the design does not make a priori assumptions about process model structure or order. SPC directly designs the controller from the recorded measurement data. The formulation of SPC neither requires the solution of a non-linear Riccati equation, as is the case with linear quadratic control, nor the solution of a recursive Diophantine equation, as with generalised predictive control. The realization of SPC is through singular value and QR decompositions, which makes the algorithm numerically stable and computationally efficient.

The goal of this paper is to propose a Gain Scheduled Subspace Predictive control (GSSPC) design strategy for the effective control of a PWR-type NPP. Specifically, two different variants are proposed, namely Parametrized GSSPC and Weighted GSSPC. The main advantage of the proposed technique is that it can be used in the absence of a plant model. GSSPC can be employed particularly when process knowledge is restricted to measurements at few equilibrium points. The scheduling variables are chosen to indicate variations in process behaviour as operating levels change. The computational complexity of the proposed approach is much less than other non-linear control design approaches. Performance of the proposed technique is evaluated for demand set-point variations during the load-following mode of operation. The controller is effectively able to handle ramp and step variations in the desired power.

The remainder of the paper is arranged as follows. Section II formulates the control design problem. Section III formulates the proposed GSSPC strategy. Section IV evaluates the proposed control technique on a simulated PWR-type nuclear reactor and discusses its efficacy through load-following transients. Finally, conclusions are drawn in Section V indicating the main contributions.

\section{Problem Formulation}

Consider a non-linear process which can be written by a family of linear systems in a parameter-varying innovation form at different equilibrium points as,

$$
\begin{aligned}
x[k+1] & =A_{\sigma_{i}} x[k]+B_{\sigma_{i}} u[k]+K_{\sigma_{i}} e[k], \\
y[k] & =C_{\sigma_{i}} x[k]+e[k], \quad i=1,2, \ldots, S
\end{aligned}
$$

where the state is $x[k] \in \mathbb{R}^{n}$, the input is $u[k] \in \mathbb{R}^{m}$, the output is $y[k] \in \mathbb{R}^{l}$, and the innovation sequence is $e[k] \in \mathbb{R}^{l}$, with the error covariance matrix $E\left(e[k] e^{T}[k]\right)=S . A_{\sigma_{i}} \in$ $\mathbb{R}^{n \times n}, B_{\sigma_{i}} \in \mathbb{R}^{n \times m}, K_{\sigma_{i}} \in \mathbb{R}^{n \times l}$, and $C_{\sigma_{i}} \in \mathbb{R}^{l \times n}$ are system matrices. $\sigma_{i}$ and $S$ denote equilibrium point and the total number of equilibrium points, respectively.

The problem is to design a control input $\left(\mathbf{u}_{\mathbf{f}}\right)$, such that the predicted output $\left(\hat{\mathbf{y}}_{\mathbf{f}}\right)$ tracks a reference signal $\left(\mathbf{r}_{\mathbf{f}}\right)$. The control input can be computed by minimizing the cost function given by

$$
J=\min _{\Delta \mathbf{u}_{\mathbf{f}}}\left(\left(\hat{\mathbf{y}}_{\mathbf{f}}-\mathbf{r}_{\mathbf{f}}\right)^{T} Q_{f}\left(\hat{\mathbf{y}}_{\mathbf{f}}-\mathbf{r}_{\mathbf{f}}\right)+\Delta \mathbf{u}_{\mathbf{f}}^{T} R_{f} \Delta \mathbf{u}_{\mathbf{f}}\right),
$$

where

$\mathbf{r}_{\mathbf{f}}=\left[\begin{array}{llll}r^{T}[t+1] & r^{T}[t+2] & \cdots & r^{T}\left[t+N_{p}\right]\end{array}\right]^{T}$, $\hat{\mathbf{y}}_{\mathbf{f}}=\left[\begin{array}{llll}\hat{y}^{T}[t+1] & \hat{y}^{T}[t+2] & \cdots & \hat{y}^{T}\left[t+N_{p}\right]\end{array}\right]^{T}$, $\mathbf{u}_{\mathbf{f}}=\left[\begin{array}{llll}u^{T}[t+1] & u^{T}[t+2] & \cdots & u^{T}\left[t+N_{c}\right]\end{array}\right]^{T}$.

$N_{c}\left(\leq N_{p}\right)$ and $N_{p}$ are control and prediction horizons, respectively. $R_{f}=I_{N_{c}} \otimes R$ penalizes the rate of change of input, where $R \in \mathbb{R}^{m \times m}$ is a positive definite matrix, $I_{N_{c}}$ is an $N_{c} \times N_{c}$ identity matrix, and $\otimes$ denotes the Kronecker product. Similarly, $Q_{f}=I_{N_{p}} \otimes Q$ penalizes the error between desired reference and output, where $Q \in \mathbb{R}^{l \times l}$ is a positive semi-definite matrix.

\section{Proposed CONTROL ApProACH}

\section{A. Subspace Predictor}

The formulation of SPC requires the design of a predictor to compute the control law. A set of block Hankel matrices can be formed from the collected measurement data at different equilibrium points. They are given as

$$
\begin{aligned}
Y_{P}= & {\left.\left[\begin{array}{cccc}
y[1] & y[2] & \cdots & y[N-2 f+1] \\
y[2] & y[3] & \cdots & y[N-2 f+2] \\
\vdots & \vdots & \ddots & \vdots \\
y[f] & y[f+1] & \cdots & y[N-f]
\end{array}\right] ; \quad 3\right) } \\
Y_{F}= & {\left[\begin{array}{cccc}
y[f+1] & y[f+2] & \cdots & y[N-f+1] \\
y[f+2] & y[f+3] & \cdots & y[N-f+2] \\
\vdots & \vdots & \ddots & \vdots \\
y[2 f] & y[2 f+1] & \cdots & y[N]
\end{array}\right] \text { (4) } }
\end{aligned}
$$

where $f$ is the order of predictor matrix. $Y_{P} \in$ $\mathbb{R}^{f l \times(N-2 f+1)}$ and $Y_{F} \in \mathbb{R}^{f l \times(N-2 f+1)}$ are called as past and future output data Hankel matrices, respectively. Similarly, $U_{P} \in \mathbb{R}^{f m \times(N-2 f+1)}, E_{P} \in \mathbb{R}^{f l \times(N-2 f+1)}$, and $X_{P} \in \mathbb{R}^{n \times(N-2 f+1)}$ are defined as past input, past innovation, and past state matrices, respectively. The same notation holds true with subscript $F$ terms to define future Hankel matrices. Using these definitions,

$$
\begin{aligned}
& Y_{P}=\Gamma_{\sigma_{i}, f} X_{P}+H_{\sigma_{i}, f}^{d} U_{P}+H_{\sigma_{i}, f}^{s} E_{P}, \\
& Y_{F}=\Gamma_{\sigma_{i}, f} X_{F}+H_{\sigma_{i}, f}^{d} U_{F}+H_{\sigma_{i}, f}^{s} E_{F}, \\
& X_{F}=A_{\sigma_{i}}^{f} X_{P}+\Delta_{\sigma_{i}, f}^{d} U_{P}+\Delta_{\sigma_{i}, f}^{s} E_{P},
\end{aligned}
$$

where $\Gamma_{\sigma_{i}, f} \in \mathbb{R}^{f l \times n}$ is the extended observability matrix. $H_{\sigma_{i}, f}^{d} \in \mathbb{R}^{f l \times f m}$ and $H_{\sigma_{i}, f}^{s} \in \mathbb{R}^{f l \times f l}$ are deterministic and stochastic lower block-triangular Toeplitz matrices, respectively. $\Delta_{\sigma_{i}, f}^{d} \in \mathbb{R}^{n \times f m}$ and $\Delta_{\sigma_{i}, f}^{s} \in \mathbb{R}^{n \times f l}$ are deterministic and stochastic reverse extended controllability matrices, respectively. Thus, from (6), the predictor is given 
by

$$
\hat{Y}_{F}=L_{\sigma_{i}, w} W_{P}+L_{\sigma_{i}, u} U_{F},
$$

where $W_{P}=\left[\begin{array}{cc}Y_{P}^{T} & U_{P}^{T}\end{array}\right]^{T} \in \mathbb{R}^{f(m+l) \times(N-2 f+1)}$. $L_{\sigma_{i}, w} \in \mathbb{R}^{f l \times f(m+l)}$ and $L_{\sigma_{i}, u} \in \mathbb{R}^{f l \times f m}$ are predictor matrices at different equilibrium points.

\section{B. Subspace Predictive Control}

In order to incorporate the above defined predictor in SPC, it is sufficient to consider only the leftmost column of $\hat{Y}_{f}$. Thus, (6) can be rewritten as

$$
\hat{\mathbf{y}}_{\mathbf{f}}=L_{\sigma_{i}, w} \mathbf{w}_{\mathbf{p}}+L_{\sigma_{i}, u} \mathbf{u}_{\mathbf{f}},
$$

or simply in terms of input increments as,

$$
\hat{\mathbf{y}}_{\mathbf{f}}=\overline{\mathrm{I}}_{l} y[k]+O_{l} L_{\sigma_{i}, w} \Delta \mathbf{w}_{\mathbf{p}}+O_{l} L_{\sigma_{i}, u} \Delta \mathbf{u}_{\mathbf{f}}
$$

where

$O_{l}=\left[\begin{array}{cccc}I_{l} & 0 & \cdots & 0 \\ I_{l} & I_{l} & \cdots & 0 \\ \vdots & \vdots & \ddots & \vdots \\ I_{l} & I_{l} & \cdots & I_{l}\end{array}\right] \in R^{N_{p} l \times N_{p} l}, \overline{\mathrm{I}}_{l}=\left[\begin{array}{c}I_{l} \\ I_{l} \\ \vdots \\ I_{l}\end{array}\right] \in R^{N_{p} l \times l}$

and $I_{l}$ is an $l \times l$ identity matrix. Rewriting (8), by using $\overline{\mathbf{y}}[k]=\overline{\mathrm{I}}_{l} y[k], \bar{L}_{\sigma_{i}, w}=O_{l} L_{\sigma_{i}, w}$, and $\bar{L}_{\sigma_{i}, u}=O_{l} L_{\sigma_{i}, u}$, as

$$
\hat{\mathbf{y}}_{\mathbf{f}}=\overline{\mathbf{y}}[k]+\bar{L}_{\sigma_{i}, w} \Delta \mathbf{w}_{\mathbf{p}}+\bar{L}_{\sigma_{i}, u} \Delta \mathbf{u}_{\mathbf{f}} .
$$

For the case of unconstrained SPC design, the input increment can be computed as

$\Delta \mathbf{u}_{\mathbf{f}}=-\left(\left(\bar{L}_{\sigma_{i}, u}\right)^{T} Q_{f} \bar{L}_{\sigma_{i}, u}+R_{f}\right)^{-1}\left(\bar{L}_{\sigma_{i}, u}\right)^{T} Q_{f}\left(\overline{\mathbf{y}}[k]-\mathbf{r}_{\mathbf{f}}+\bar{L}_{\sigma_{i}, w} \Delta \mathbf{w}_{\mathbf{p}}\right)$,

or simply,

$$
\Delta \mathbf{u}_{\mathbf{f}}=-K_{\sigma_{i}, u}\left(\overline{\mathbf{y}}[k]-\mathbf{r}_{\mathbf{f}}\right)-K_{\sigma_{i}, w} \Delta \mathbf{w}_{\mathbf{p}}
$$

where the gain matrices are defined by,

$$
\begin{aligned}
& K_{\sigma_{i}, u}=\left(\left(\bar{L}_{\sigma_{i}, u}\right)^{T} Q_{f} \bar{L}_{\sigma_{i}, u}+R_{f}\right)^{-1}\left(\bar{L}_{\sigma_{i}, u}\right)^{T} Q_{f}, \\
& K_{\sigma_{i}, w}=\left(\left(\bar{L}_{\sigma_{i}, u}\right)^{T} Q_{f} \bar{L}_{\sigma_{i}, u}+R_{f}\right)^{-1}\left(\bar{L}_{\sigma_{i}, u}\right)^{T} Q_{f} \bar{L}_{\sigma_{i}, w} .
\end{aligned}
$$

Finally, the control signal is updated using only the first element of the control move

$$
u[t+1]=\Delta \mathbf{u}_{\mathbf{f}}[1]+u[k] .
$$

\section{Gain-Scheduled SPC}

Gain scheduling is widely employed in the control of nonlinear processes where an approximate relationship between the plant dynamics and operating conditions is known. Gain scheduled SPC can be described as a two-step procedure. The first step is to formulate linear subspace predictors for the process from measurements. This design process calculates a set of predictor matrices corresponding to the process at different equilibrium points. The second step involves implementing the family of linear controllers such that the controller parameters are scheduled according to the current value of the scheduling variables. Scheduling variables indicate the closeness of system behaviour to an operating point.
Control input, reference input, plant output, etc., are usually used as scheduling variables. In this work, the scheduling strategy for the control loop took into account the reference input. Here, two different approaches are proposed for the second step and are discussed as follows. The block diagram of the proposed technique is shown in Fig. 1.

1) Parametrized GSSPC: The SPC controllers designed at different operating points are parametrized and integrated to form a family of controllers. They are parametrized according to the preprogrammed adjustment schedule due to significant changes in scheduling variables. Thus, the resulting controller is implemented as a single control law, whose parameters are varied following the scheduling variables. The interpolation strategy is employed to arrive at a family of controllers parameters from a set of predictor designs at isolated values of the equilibrium points. The algorithm works by using a controller as long as the system state is close to the corresponding operating point. Based on the switching parameter, it then switches to the next controller when the system state is sufficiently close to the next operating point. The algorithm easily combines two or more controllers using linear interpolation. The linear interpolation in the interval $\sigma_{i} \leq \sigma \leq \sigma_{i+1}$ can be denoted as

$$
\begin{aligned}
K_{\sigma, u} & =\frac{\sigma-\sigma_{i+1}}{\sigma_{i}-\sigma_{i+1}} K_{\sigma_{i}, u}+\frac{\sigma-\sigma_{i+1}}{\sigma_{i+1}-\sigma_{i}} K_{\sigma_{i+1}, u} \\
K_{\sigma, w} & =\frac{\sigma-\sigma_{i+1}}{\sigma_{i}-\sigma_{i+1}} K_{\sigma_{i}, w}+\frac{\sigma-\sigma_{i+1}}{\sigma_{i+1}-\sigma_{i}} K_{\sigma_{i+1}, w}
\end{aligned}
$$

where $\sigma_{i}$ and $\sigma_{i+1}$ represent operating points.

2) Weighted GSSPC: A weighing mechanism is employed to use weighted combination of the outputs of the individual controllers. The control output of SPC controllers designed at different operating points are weighed in accordance to the scheduling variable. If the system state is close to an operating point, then the corresponding control output will dominate in the control signal while outputs from rest of the controllers are weighed less. Hence, the overall control input is a combination of control inputs. It is given by

$$
u[k]=\sum_{i=1}^{S} W_{\sigma_{i}} u_{\sigma_{i}}[k]
$$

where $W_{\sigma_{i}}$ is the weight corresponding to the operating point $\sigma_{i}$ and satisfies $\sum_{i=1}^{S} W_{\sigma_{i}}=1 . u_{\sigma_{i}}$ is the control signal from the controller corresponding to $\sigma_{i}$ th operating point. It is to be noted that the weighted GSSPC approach combines weighted controller outputs whereas, parametrized GSSPC employs linear controller interpolation strategy.

\section{Performance Assessment}

The control performance can be evaluated based on the following factors: Percentage root mean squared error (PRMSE) computed between output and demand set-point analyses the effect of control action on the output. The effect of control action on input is analysed by computing the total variation of input (TVI) and the L2-norm of input $\left(\|U\|_{2}\right)$. These are 


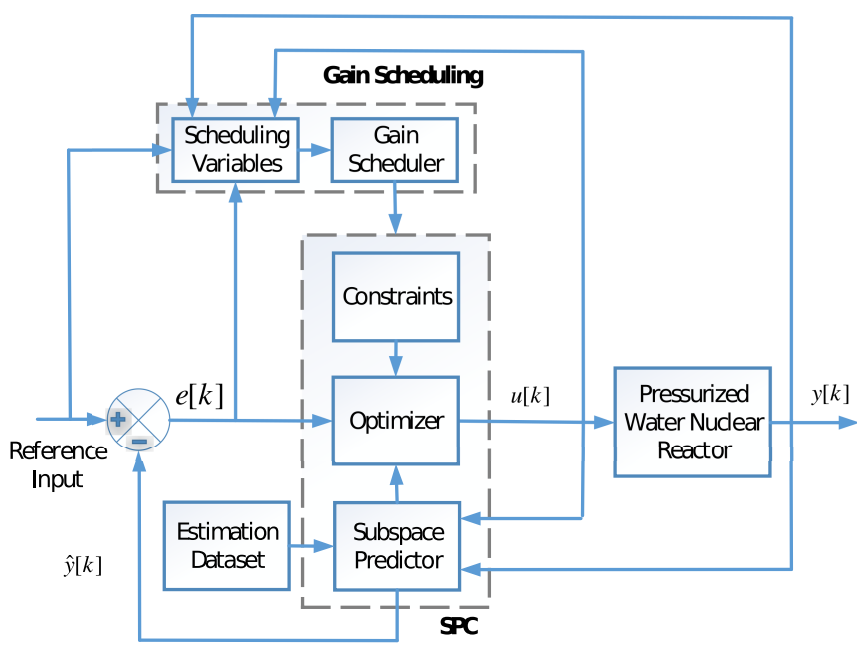

Fig. 1. Block diagram of the proposed GSSPC algorithm.

given by,

$$
\begin{aligned}
\text { PRMSE } & =\frac{1}{N} \sqrt{\sum_{k=1}^{N}(y[k]-r[k])^{2}} \times 100 \%, \\
T V I & =\sum_{k=1}^{N}|(u[k+1]-u[k])| \\
\|U\|_{2} & =\sqrt{\left(\sum_{k=1}^{N}(u[k])^{2}\right)} .
\end{aligned}
$$

\section{Case Study: Application to a Simulated PWR-TYPE NUCLEAR REACTOR}

For identification and control, the dynamical model of a PWR can be suitably defined using the point kinetics equation with six groups of delayed neutrons precursors' concentration coupled with thermal-hydraulics. The model is based on the following assumptions: The primary loop is characterized by a lumped model. The pressure and mass flow rate are constants. The heat generated in the core is transferred using a single phase coolant [2], [18].

The primary loop model equations are as follows:

$$
\begin{aligned}
\frac{d P}{d t} & =\frac{\rho_{T}-\sum_{j=1}^{6} \beta_{j}}{\Lambda} P+\frac{\sum_{j=1}^{6} \beta_{j} C_{j}}{\Lambda}, \\
\frac{d C_{j}}{d t} & =\lambda_{j}\left(P-C_{j}\right), \quad j=1,2, \ldots, 6, \\
\frac{d T_{f}}{d t} & =\frac{P}{\mu_{f}}-\frac{\Omega}{\mu_{f}}\left(T_{f}-T_{c}\right), \\
\frac{d T_{c}}{d t} & =\frac{\Omega}{\mu_{c}}\left(T_{f}-T_{c}\right)-\frac{M_{c}}{\mu_{c}}\left(T_{\text {out }}-T_{\text {in }}\right), \\
T_{c} & =\frac{T_{\text {out }}+T_{\text {in }}}{2}, \\
\rho_{T} & =\rho+\alpha_{f} T_{f}+\alpha_{c} T_{c} .
\end{aligned}
$$

where $P$ is normalized neutronic power; $\beta_{j}, \lambda_{j}$, and $C_{j}$ denote fraction of delayed neutrons, decay constant, and
TABLE I

REACTOR PARAMETERS

\begin{tabular}{|c|c|c|c|c|c|c|}
\hline Group, $\mathrm{i}$ & 1 & 2 & 3 & 4 & 5 & 6 \\
\hline$\lambda_{i}\left(s^{-1}\right)$ & 0.0125 & 0.0308 & 0.1152 & 0.3109 & 1.240 & 3.3287 \\
\hline$\beta_{i}$ & 0.000216 & 0.001416 & 0.001349 & 0.00218 & 0.00095 & 0.000322 \\
\hline$\Lambda(s)$ & $\tau_{\text {cold }}(s)$ & $\tau_{\text {hot }}(s)$ & $\tau_{\text {sg }}(s)$ & $D_{1}\left({ }^{\circ} \mathrm{Cs}^{-1}\right)$ & $D_{2}$ & $D_{3}$ \\
$5 \times 10^{-4}$ & 7.0 & 5.0 & 11.3 & 3.746 & 0.7005 & -0.2995 \\
\hline
\end{tabular}

delayed neutron precursors' concentration of $j^{\text {th }}$ group, respectively; $\Lambda$ represents prompt neutron life time; $\rho$ and $\rho_{T}$ denote reactivity contributed by control input and the total reactivity, respectively; $T_{f}$ and $T_{c}$ are temperatures of fuel and coolant, respectively; $\alpha_{f}$ and $\alpha_{c}$ are temperature coefficients of reactivity of fuel and coolant, respectively; $H_{f}, H_{c}, \gamma_{f}$, and $\gamma_{c}$ are proportionality constants; The dynamic equations governing the secondary side are given by

$$
\begin{aligned}
\frac{d T_{\text {in }}}{d t} & =\frac{1}{\tau_{\text {cold }}}\left(D_{2} T_{s g}-D_{3} T_{\text {hot }}-T_{\text {in }}\right), \\
\frac{d T_{\text {hot }}}{d t} & =\frac{1}{\tau_{\text {hot }}}\left(T_{\text {out }}-T_{\text {hot }}\right), \\
\frac{d T_{s g}}{d t} & =-\frac{1}{\tau_{s g}}\left(T_{s g}-T_{\text {hot }}\right)-D_{1} L_{T},
\end{aligned}
$$

where $T_{\text {out }}, T_{i n}, T_{h o t}$, and $T_{s g}$ are temperatures of coreoutlet, core-inlet, hot leg and steam generator respectively. $\tau_{\text {cold }}, \tau_{\text {hot }}$, and $\tau_{s g}$ are time constants; $D_{1}, D_{2}$, and $D_{3}$ are constants; $L_{T}$ is turbine load. Values of various parameters used in (21)-(29) are listed in Tables I and II [3], [18].

The dynamic model of a nuclear reactor is perturbed by a regulating rod (RR) movement to inject reactivity into the system. A set of estimation datasets are constructed at different operating power levels in which reactivity and neutronic power (with AWGN) act as input and output, respectively. The estimation data are used for designing the subspace predictor and estimating predictor coefficients at different operating levels. The control input trajectory is calculated by solving the optimization problem. It is computed over the control horizon while only the first control action is executed and subsequently given to the process for tracking the set-point. The same steps are repeated for subsequent data samples.

In this paper, five different operating points based on the neutronic power level are considered due to availability of reactor data at these points [3]. Five different estimation dataset comprises of input reactivity and corresponding output power mixed with AWGN are constructed to estimate the parameters of controllers directly from measurements.

To validate the controller design efficacy, a typical load- 
TABLE II

REACTOR PARAMETERS AT DIFFERENT POWER LEVELS

\begin{tabular}{|c|c|c|c|c|c|c|c|}
\hline$P$ & $T_{c}\left({ }^{\circ} \mathrm{C}\right)$ & $\alpha_{f}\left({ }^{\circ} \mathrm{C}^{-1}\right)$ & $\alpha_{c}\left({ }^{\circ} \mathrm{C}^{-1}\right)$ & $\mu_{f}\left(\mathrm{~J} /{ }^{\circ} \mathrm{C}^{-1}\right)$ & $\mu_{c}\left(\mathrm{~J} /{ }^{\circ} \mathrm{C}^{-1}\right)$ & $\Omega\left(\mathrm{J} / \mathrm{s}^{\circ} \mathrm{C}^{-1}\right)$ & $M_{c}\left(\mathrm{~J} / \mathrm{s}^{\circ} \mathrm{C}^{-1}\right)$ \\
\hline 1 & 302.0 & $-2.9 \times 10^{-5}$ & $-6.30 \times 10^{-4}$ & $2.25 \times 10^{7}$ & $6.90 \times 10^{7}$ & $3.94 \times 10^{6}$ & $7.08 \times 10^{7}$ \\
\hline 0.8 & 298.6 & $-3.2 \times 10^{-5}$ & $-5.59 \times 10^{-4}$ & $2.21 \times 10^{7}$ & $6.80 \times 10^{7}$ & $4.16 \times 10^{6}$ & $6.88 \times 10^{7}$ \\
\hline 0.6 & 295.0 & $-3.3 \times 10^{-5}$ & $-5.56 \times 10^{-4}$ & $2.18 \times 10^{7}$ & $6.70 \times 10^{7}$ & $4.38 \times 10^{6}$ & $6.87 \times 10^{7}$ \\
\hline 0.4 & 291.8 & $-3.5 \times 10^{-5}$ & $-5.22 \times 10^{-4}$ & $2.14 \times 10^{7}$ & $6.61 \times 10^{7}$ & $4.61 \times 10^{6}$ & $6.79 \times 10^{7}$ \\
\hline 0.2 & 288.4 & $-3.8 \times 10^{-5}$ & $-4.86 \times 10^{-4}$ & $2.10 \times 10^{7}$ & $6.53 \times 10^{7}$ & $4.85 \times 10^{6}$ & $6.70 \times 10^{7}$ \\
\hline
\end{tabular}
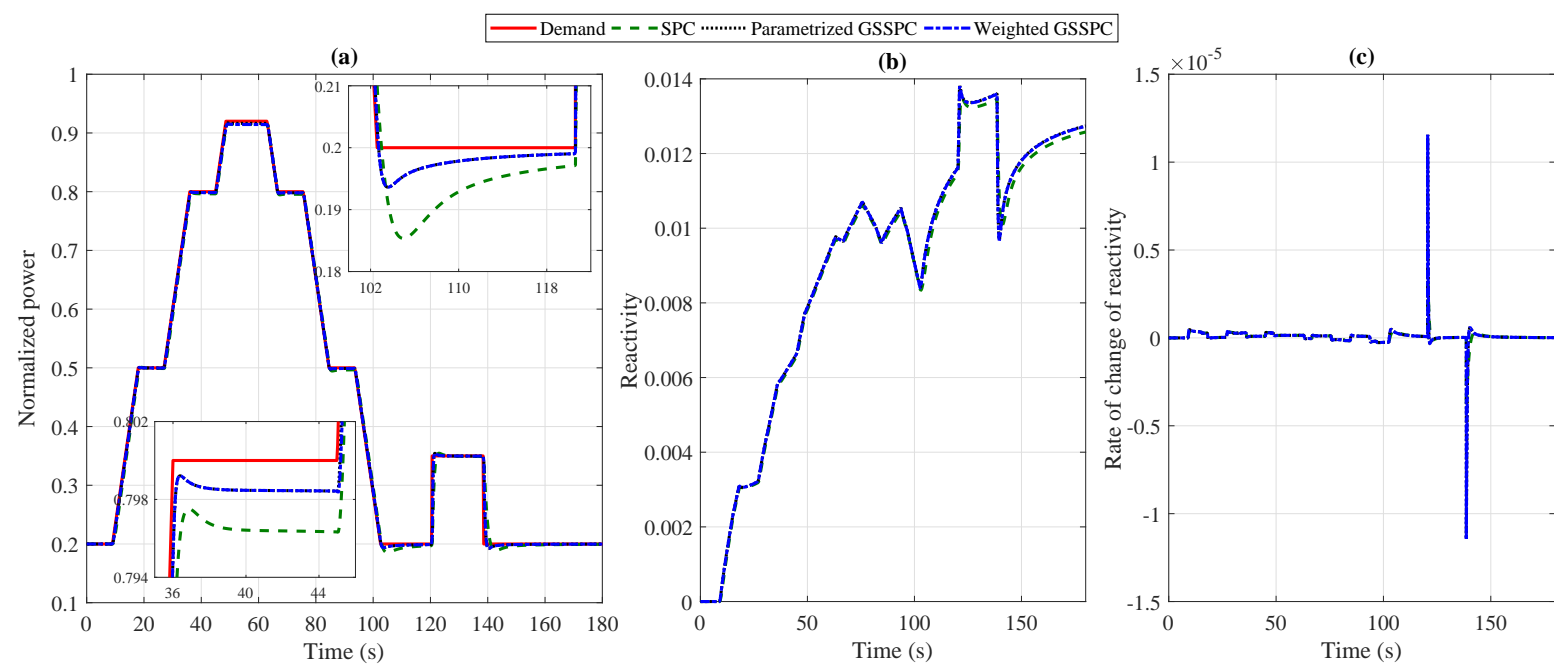

Fig. 2. Variation of (a) reactor power, (b) control input, and (c) rate of change of control input for different approaches.

following transient is considered as follows:

$$
P= \begin{cases}0.20 P, & 0 s \leq t \leq 9 s \\ 1.0(t-9) / 30+0.20, & 9 s<t \leq 18 s \\ 0.50 P, & 18 s<t \leq 27 s \\ 1.0(t-27) / 30+0.50, & 27 s<t \leq 36 s \\ 0.80 P, & 36 s<t \leq 45 s \\ 1.0(t-45) / 30+0.80, & 45 s<t \leq 48.6 s \\ 0.92 P, & 48.6 s<t \leq 63 s \\ -1.0(t-63) / 30+0.92, & 63 s<t \leq 66.6 \\ 0.80 P, & 66.6 s<t \leq 75.6 s \\ -1.0(t-75.6) / 30+0.50, & 75.6 s<t \leq 84.6 s \\ 0.50 P, & 84.6<t \leq 93.6 s \\ -1.0(t-93.6) / 30+0.50, & 93.6 s<t \leq 102.6 s \\ 0.20 P, & 102.6 s<t \leq 120.6 s \\ 0.35 P, & 120.6 s<t \leq 138.6 s \\ 0.20 P, & 111.6 s<t \leq 180 s\end{cases}
$$

Performance of the proposed controller for tracking the load cycle is shown in Fig. 2. The controller output is smoothly able to track fast varying ramp of $3.33 \% / \mathrm{s}$ and $15 \%$ step variation in the demand power as shown in Fig. 2(a). Variations of control signal and rate of change of control signal are shown in Figs. 2(b) and 2(c), respectively. Performance of proposed GSSPCs are compared with the classical SPC. GSSPCs track the demand variation very closely and with less peak overshoot. The control efforts remain approximately the same.

Another load following transient is considered to test the performance in the case of fast variations in the load. It is given as follows:

$$
P=\left\{\begin{array}{l}
1.0 P, 0 s \leq t \leq 18 s \quad \& 288 s<t \leq 360 s \\
0.9 P, 18 s<t \leq 36 s \quad \& 270 s<t \leq 288 s \\
0.8 P, 36 s<t \leq 54 s \quad \& 252 s<t \leq 270 s \\
0.7 P, 54 s<t \leq 72 s \quad \& 234 s<t \leq 252 s \\
0.6 P, 72 s<t \leq 90 s \quad \& 216 s<t \leq 234 s(31) \\
0.5 P, 90 s<t \leq 108 s \& 198 s<t \leq 216 s \\
0.4 P, 108 s<t \leq 126 s \& 170 s<t \leq 198 s \\
0.3 P, 126 s<t \leq 144 s \& 162 s<t \leq 170 s \\
0.2 P, 144 s<t \leq 162 s
\end{array}\right.
$$

Fig. 3 depicts the performance of the proposed controller for tracking the load cycle. The controller output is able to track the $10 \%$ step variation in the set-point as shown in Fig. 3(a). Variations of the control signal and the rate of change of control signal are shown in Figs. 3(b) and 3(c), respectively. From both the simulation studies it can be seen that the proposed GSSPCs can track load variation better than the classical SPC.

A quantitative performance analysis is performed by comparing performance measures, PRMSE, TVI, and $\|U\|_{2}$. Table III compares the control performances of different approaches. It is found that the PRMSE for GSSPC approaches is less than that of the classical SPC approach whereas the TVI and the $\|U\|_{2}$ are almost equal. Thus, it can be concluded that the parametrized GSSPC can track the demand variation slightly better than the weighted GSSPC. 

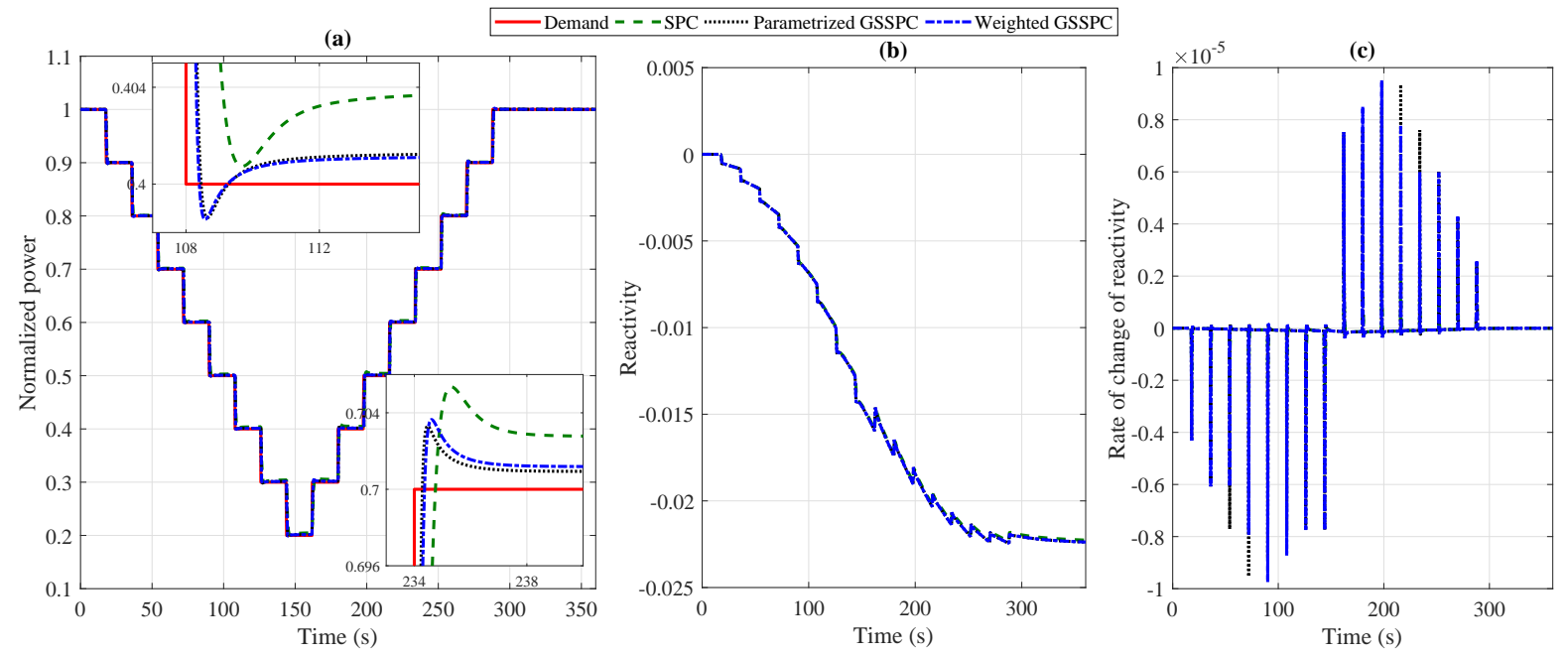

Fig. 3. Variation of (a) reactor power, (b) control input, and (c) rate of change of control input for different approaches.

TABLE III

COMPARISON OF PERFORMANCES OF DIFFERENT APPROACHES

\begin{tabular}{|c|c|c|c|c|}
\hline Case & Technique & PRMSE & TVI & $\|U\|_{2}$ \\
\hline \multirow{2}{*}{ I } & Classical SPC & $2.8124 \times 10^{-3}$ & $2.6453 \times 10^{-2}$ & 4.0962 \\
\cline { 2 - 5 } & Parametrized GSSPC & $1.6214 \times 10^{-3}$ & $2.8277 \times 10^{-2}$ & 4.1454 \\
\cline { 2 - 5 } & Weighted GSSPC & $1.6538 \times 10^{-3}$ & $2.8259 \times 10^{-2}$ & 4.1422 \\
\hline \multirow{2}{*}{ II } & Classical SPC & $1.6176 \times 10^{-3}$ & $3.3608 \times 10^{-2}$ & 9.7282 \\
\cline { 2 - 5 } & Parametrized GSSPC & $1.0519 \times 10^{-3}$ & $3.6692 \times 10^{-2}$ & 9.7804 \\
\cline { 2 - 5 } & Weighted GSSPC & $1.0587 \times 10^{-3}$ & $3.6644 \times 10^{-2}$ & 9.7800 \\
\hline
\end{tabular}

\section{CONCLUSION}

Gain scheduled subspace-based predictive control strategy that can incorporate constraints and optimize control performance has been investigated for the PWR-type nuclear reactor. Two different variants of GSSPC namely, parametrized GSSPC and weighted GSSPC are proposed in this work. The control strategy is directly designed from the recorded input-output dataset and does not require any system model a priori. Detailed simulation studies verify the performance of the proposed algorithm in controlling a parameter-varying non-linear PWR system. Proposed controllers follow the setpoint variation in the load very closely and with less peak overshoot. Comparison with classical SPC approach shows the effectiveness of proposed controllers. The designed controllers are adequately able to handle different fast varying ramp and step variations in the load.

\section{ACKNOWLEDGEMENT}

The work presented in this paper has been financially supported under grants EP/R021961/1 and EP/R022062/1 from the Engineering and Physical Sciences Research Council.

\section{REFERENCES}

[1] S. J. Qin and T. A. Badgwell, "A survey of industrial model predictive control technology," Control Engineering Practice, vol. 11, no. 7, pp. 733-764, 2003.

[2] M. G. Na, D. W. Jung, S. H. Shin, J. W. Jang, K. B. Lee, and Y. J. Lee, "A model predictive controller for load-following operation of PWR reactors," IEEE Transactions on Nuclear Science, vol. 52, no. 4, pp. 1009-1020, Aug 2005.
[3] T. Yun, H. Su-xia, L. Chong, and Z. Fu-yu, "An improved implicit multiple model predictive control used for movable nuclear power plant," Nuclear Engineering Design, vol. 240, pp. 3582-3585, 2010.

[4] H. Eliasi, M. B. Menhaj, and H. Davilu, "Robust nonlinear model predictive control for nuclear power plants in load following operations with bounded xenon oscillations," Nuclear Engineering Design, vol. 241, pp. 533-543, 2011.

[5] G. Wang, J. Wu, B. Zeng, Z. Xu, W. Wu, and X. Ma, "Statespace model predictive control method for core power control in pressurized water reactor nuclear power stations," Nuclear Engineering Technology, vol. 49, no. 1, pp. 134-140, 2017.

[6] M. V. Kothare, B. Mettler, M. Morari, P. Bendotti, and C.-M. Falinower, "Linear parameter varying model predictive contr for steam generator level control," Computers \& Chemical Engineering, vol. 21, pp. S861-S866, may 1997.

[7] W. J. Rugh and J. S. Shamma, "Research on gain scheduling," Automatica, vol. 36, no. 10, pp. 1401 - 1425, 2000.

[8] L. Lemazurier, M. Yagoubi, P. Chevrel, and A. Grossetłte, "Multiobjective h2/h gain-scheduled nuclear core control design," IFACPapersOnLine, vol. 50, no. 1, pp. 3256 - 3262, 2017.

[9] A. Alsharkawi and J. A. Rossiter, "Towards an improved gain scheduling predictive control strategy for a solar thermal power plant," IET Control Theory \& Applications, vol. 11, no. 12, pp. 1938-1947, Aug 2017.

[10] G. R. Ansarifar, H. Davilu, and H. A. Talebi, "Gain scheduled dynamic sliding mode control for nuclear steam generators," Progress in Nuclear Energy, vol. 53, no. 6, pp. 651 - 663, 2011.

[11] J. Wang, J. Zhou, X. Luan, and Z. Yang, "Discrete gain-scheduled PD control to nuclear steam generator water level," in 2016 35th Chinese Control Conference (CCC), July 2016, pp. 2992-2997.

[12] — , "L1 gain scheduled adaptive control to water level of nuclear steam generator," in 2019 IEEE International Conference on Mechatronics and Automation (ICMA), Aug 2019, pp. 1206-1211.

[13] W. Favoreel and B. D. Moor, "SPC: Subspace predictive control," in Proceedings of IFAC World Congress, Beijing, 1998, pp. 235-240.

[14] R. Kadali, "A data driven subspace approach to predictive controller design," Control Engineering Practice, vol. 11, no. 3, pp. 261-278, 2003.

[15] X. Wu, J. Shen, Y. Li, and K. Y. Lee, "Data-driven modeling and predictive control for boiler-turbine unit," IEEE Transaction on Energy Conversion, vol. 28, no. 3, pp. 470-481, Sep 2013.

[16] V. Vajpayee, S. Mukhopadhyay, and A. P. Tiwari, "Subspace-based wavelet preprocessed data-driven predictive control," INCOSE International Symposium, vol. 26, no. s1, pp. 357-371, 2016.

[17] S. Sedghizadeh and S. Beheshti, "Particle swarm optimization based fuzzy gain scheduled subspace predictive control," Engineering Applications of Artificial Intelligence, vol. 67, pp. 331 - 344, 2018.

[18] V. Vajpayee, S. Mukhopadhyay, and A. P. Tiwari, "Data-driven subspace predictive control of a nuclear reactor," IEEE Transactions on Nuclear Science, vol. 65, no. 2, pp. 666-679, Feb 2018. 